\title{
DNA Gyrase and Topoisomerase IV Mutations and their effect on Quinolones Resistant Proteus mirabilis among UTIs Patients
}

\author{
Randa H Abdelkreem ${ }^{1}$, Amjad M Yousuf ${ }^{2}$, \\ Miskelyemen A. Elmekki ${ }^{3}$, Mogahid M Elhassan ${ }^{4}$
}

ABSTRACT

Objective: This study aimed to highlight the importance of mutations within Proteus mirabilis genome that are related to fluoroquinolone resistance.

Methods: This is a cross sectional study performed in different teaching hospitals in Khartoum State from June 2016 to May 2017. A total of (120) P mirabilis isolates from patients with symptoms of UTIs attending different hospitals in Khartoum State were examined. First, modified Kurby Bauer method was performed for phenotypical detection of resistant isolates. Then polymerase chain reaction-restriction fragment length polymorphism (PCR-RFLP) followed by sequencing were applied for detection of mutations in GyrA, GyrB, ParC and ParE genes of isolates.

Results: P. mirabilis showed $30 \%$ resistance to ciprofloxacin. All samples revealed mutation at (serine 83 ) of GyrA and (serine 84) of ParC by Hinf1 restriction endonuclease digestion. Sequencing was performed for 12 samples. For each gene, two resistant and one susceptible strains were randomly selected. The mutations associated with ciprofloxacin resistant $P$. mirabilis were as follows; (1/3) GyrA (Ser 83 to Ile) and (2/3) ParC (Ser 81 to lle). Also it revealed silent mutations at codons of GyrB 474 leucine (3/3), 585 valine (2/3), 612 histidine (1/3) and 639 asparagine (1/3) and ParE 469 isoleucine (2/3), 531 aspartic (2/3) and 533 glycine $(1 / 3)$.

Conclusions: Ciprofloxacin resistance in $P$. mirabilis could be monitored through detection of mutations within DNA gyrase (encoded by gyrA and gyrB) and topoisomerase IV (encoded by parC and parE).

KEYWORDS: Ciprofloxacin resistance, Proteus mirabilis, Sudan.

doi: https://doi.org/10.12669/pjms.36.6.2207

How to cite this:

Abdelkreem RH, Yousuf AM, Elmekki MA, Elhassan MM. DNA Gyrase and Topoisomerase IV Mutations and their effect on Quinolones Resistant Proteus mirabilis among UTIs Patients. Pak J Med Sci. 2020;36(6):1234-1240. doi: https://doi.org/10.12669/pjms.36.6.2207

This is an Open Access article distributed under the terms of the Creative Commons Attribution License (http://creativecommons.org/licenses/by/3.0), which permits unrestricted use, distribution, and reproduction in any medium, provided the original work is properly cited.

1. Randa H Abdelkreem

Dept. of Microbiology, College of Medical Laboratory Science,

Shendi University, Shendi, Sudan.

2. Amjad M Yousuf

3. Miskelyemen A. Elmekki

4. Mogahid M Elhassan

2-4: Dept. of Medical Laboratory Technology, College of Applied Medical Sciences, Taibah University, Al-Madinah Al-Monawwarah, Saudi Arabia.

Correspondence:

Prof. Mogahid M Elhassan,

Dept. of Medical Laboratory Technology, College of Applied Medical Sciences, Taibah University, Almadinah Almonawwarah, Saudi Arabia.

E-mail: mmemam@taibahu.edu.sa mogahidelhassan@yahoo.com

* Received for Publication:

* $1^{\text {st }}$ Revision Received:

* $\quad 2^{\text {nd }}$ Revision Received:

* Final Revision Accepted:
January 9, 2020

March 28, 2020

June 27, 2020

June 30,2020

\section{INTRODUCTION}

Proteus mirabilis is a small gram-negative bacilli and a facultative anaerobe, it ferments maltose, but not lactose. Moreover, Proteus mirabilis is one of the common causes of urinary tract infections (UTIs) among Enterobacteriaceae. ${ }^{1}$

Ciprofloxacin is a recommended drug for the treatment of UTIs. ${ }^{2}$ Though wild-type strains of $\mathrm{P}$. mirabilis are usually susceptible to fluoroquinolones ${ }^{2,3}$ but a progressive increase in fluoroquinolone resistance has been seen in the clinical isolates of the bacterium recently. ${ }^{3,4}$ 
The basic mechanisms of quinolone resistance are represented by the changes in the active sites of the target enzymes DNA gyrase and topoisomerase IV. The degree of resistance of different regions (QRDRs) encoded by gyrA and parC gene mutations have been described in several studies. ${ }^{5}$ In Sudan, a recent study that analyzed the antimicrobial susceptibility patterns of several species of Gram-negative bacteria, including $P$ mirabilis, to four different groups of antibiotics showed that $(22.3 \%)$ of the isolates were resistant to three or more classes of antibiotics, including cephalosporins, $\beta$-lactam- $\beta$-lactamase inhibitor, quinolones, aminoglycosides and carbapenems. ${ }^{6}$

\section{METHODS}

The study was carried out using 120 Proteus mirabilis urinary isolates collected from different hospitals in Khartoum State. The isolates were collected during the period from June 2016 to May 2017. This study obtained ethical approval number (MLT 711/2016) from the ethical committee of SUST.

Bacteriology: Urine samples were cultured and P.mirabilis was isolated and identified by the conventional standard methods. All the grown isolates were tested for their ciprofloxacin resistance in vitro by the Kirby-Baur disk diffusion method against ciprofloxacin (CIP) $(5 \mu \mathrm{g} / \mathrm{ml}){ }^{7}$

\section{Polymerase Chain Reaction:}

DNA Extraction: DNA of Proteus mirabilis was isolated from overnight growth on nutrient agar. For each isolate, several colonies of pure culture were suspended in $(500 \mu \mathrm{L})$ of sterile deionized water in $1.5 \mathrm{ml}$ eppendorf tube for each isolate, and boiled for (10 minutes). Then tubes were centrifuged at $(14000 \mathrm{~g})$ for (10 minutes) using a microcentrifuge and supernatant was stored at $\left(-20^{\circ} \mathrm{C}\right)$ as a template DNA stock. ${ }^{8}$ The purity of the extracted DNA was determined by running the DNA sample on $(2 \%)$ agarose gel. ${ }^{9}$

Primer Design: Degenerate oligonucleotide primers (Table-I) from conserved regions of the GyrA, GyrB, ParC and ParE genes were designed by primer3plus (www.bioinformatics.nl/primer3plus) from Proteus mirabilis HI4320 DNA sequences in the Gen Bank database (NCBI) and were synthesized by Macrogen (South Korea).

Amplification of GyrA, GyrB, ParC and ParE Genes: DNA amplification was done using Maxime PCR Premix kit (I-Taq) (iNtRON, Korea) which is a lyophilized master mix. The PCR assay was carried out in a total volume of $(20 \mu \mathrm{L})$ of mixture containing $(0.5 \mu \mathrm{L})$ of each of the virulence genespecific primers $(1 \mu \mathrm{L}$ total volume for forward and reverse primer in each case), $(2 \mu \mathrm{L})$ of template DNA and $(17 \mu \mathrm{L})$ of water for injection (WFI). The amplification was done using (CLASSIC K960 China thermal cycler).

Restriction Fragment Length Polymorphism (RFLP-PCR): The PCR product was digested with HinfI restriction enzyme (CutSmart ${ }^{\mathrm{TM}}$, New England Biolabs, Inc) and endonuclease digestion was performed as recommended by the manufacturer to detect GyrA (ser 83) and ParC (ser 81) mutations. Sequencing of the Target Genes: Three products were selected randomly to detect GyrA, GyrB, ParC and ParE, Sequencing was performed in both directions with the same set of primers used for the PCR by Sanger dideoxy chain termination method.

Data and Genetic Analysis: The data was analyzed using statistical software package (SPSS - version 20). The sequences were checked for similarity with reference genes using NCBI's BLAST (http:/ / www.ncbi.nlm.nih.gov/blast). The sequences were translated into amino acid codons using Expasy translation tool. The protein sequences were then checked for similarity in BLAST.

\section{RESULTS}

\section{Bacteriological Findings:}

Culture: The identification scheme confirmed that (120) of the isolates belonged to the species $P$. mirabilis.

Disk Diffusion Method: The results of modified Kirby-Bauer method showed that $P$. mirabilis reflected relatively decreased sensitivity to ciprofloxacin as only $84(70 \%)$ of the isolates were sensitive while $36(30 \%)$ were resistant, with a statistically significant difference $(\mathrm{p}=0.000)$.

Table-I: Primers used for detection of virulence genes in Proteus mirabilis strains.

\begin{tabular}{llr}
\hline Primer & primers Sequence & $\begin{array}{r}\text { Product } \\
\text { size bp }\end{array}$ \\
\hline Gyr A & F 5' - AGCGACATTGCCAGAGAAAT -3' & 937 \\
& R 5' - CACCGACTGCATCACGTTT -3' & \\
Gyr B & F 5'- GGCAAAACAAGGGCGTAA-3' & 822 \\
& R 5' - GCCCCTTCTTCAATCAGGTT-3' & \\
Par C & F 5' - CAGCGTCGTATCGTCTATGC-3' & 992 \\
& R 5'-CGGCGTAATACTTTTTCTAAGC-3' \\
Par E & F 5'-GGAAGGAGGCGATTTACTCA-3' & 972 \\
& R 5'-GGATCAAGCGTTGTCTCACG-3' & \\
\hline
\end{tabular}




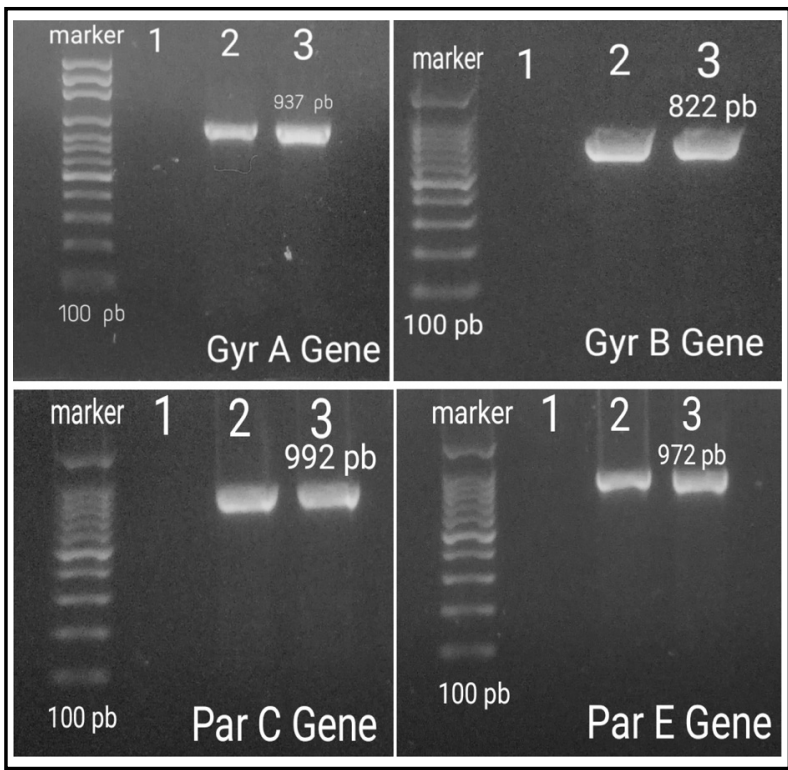

Fig.1: PCR products of GyrA, GyrB, ParC and ParE gene products on $2 \%$ agarose gel; lane 1: Negative control,

lanes 2 and 3: PCR products.

\section{Polymerase Chain Reaction (PCR):}

PCR for the amplification of GyrA, GyrB, ParC and ParE Genes: Degenerate oligonucleotide primers from conserved regions of the GyrA, GyrB, ParC and ParE genes were designed from alignments of known DNA sequences in the Gen Bank database

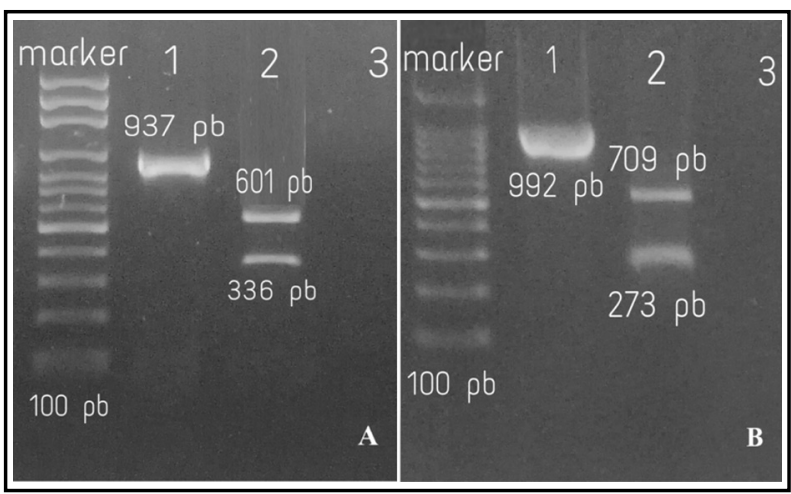

Fig.2: PCR products of GyrA and ParC were digested with HinfI and separated by $2 \%$ agarose gel. A (GyrA); lane 1: non-digested products (937 bp), lane 2: HifIdigested product (601 and $336 \mathrm{bp}$ ) lane 3: negative control and B (ParC); lane 1: non-digested products (992 bp), lane 2: HifI-digested product (709 and $273 \mathrm{bp}$ ) lane 3: negative control.

(NCBI). PCR amplification targeted GyrA gene product (937 bp) which encoded (312) amino acids, GyrB gene product (822bp) which encoded (274) amino acids, ParC gene product (992bp) which encoded (230) amino acid and ParE gene product (972bp) which encoded (324) amino acids on $P$. mirabilis as seen on $2 \%$ agarose gel (Fig.1).

Hinf Digestion of GyrA and ParC Genes: Quick screening of the (120) isolates of P.mirabilis to detect

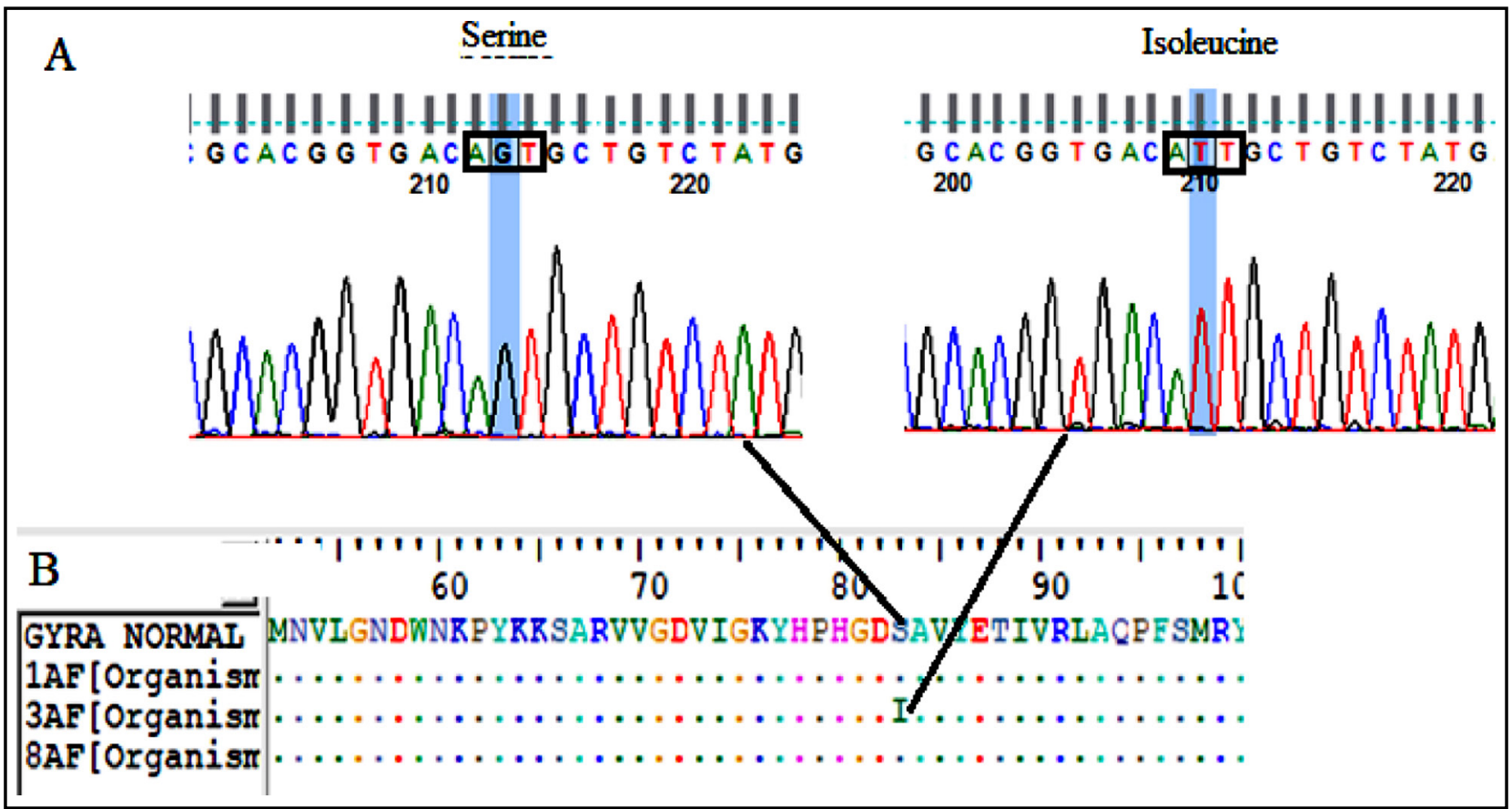

Fig.3: Line (A) Chromatograms of Sanger DNA sequencing of GyrA changed from $G$ to $T$ which change serine to isoleucine, line (B) GyrA amino acid changed codon 83 serine to isoleucine (AGT- ATT). Analyses was done by BioEdit alignment editor v7.2.5. 


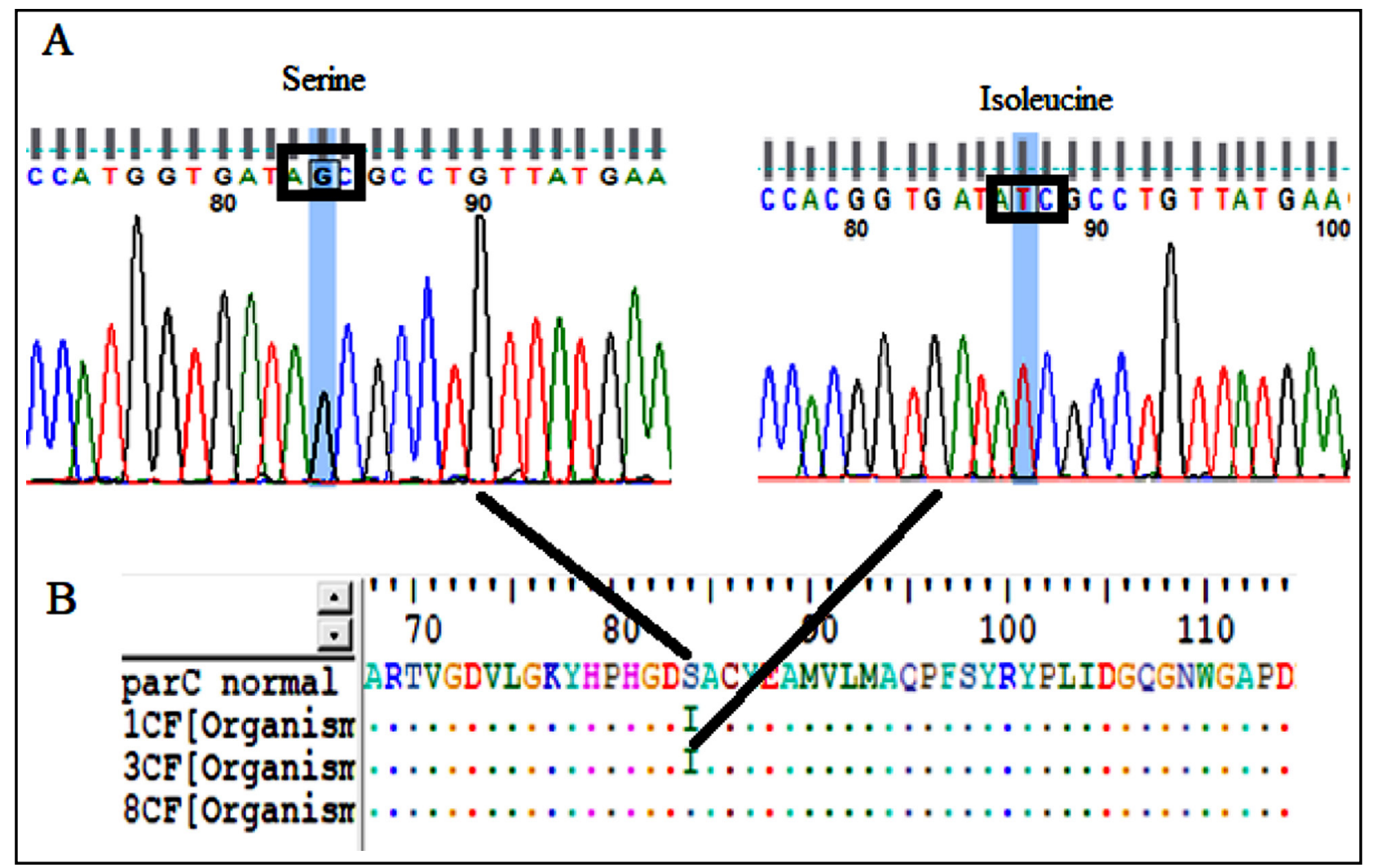

Fig.4: Line (A) Chromatograms of Sanger DNA sequencing of ParC serine 84 changed from G to T isoleucine, line (B) ParC amino acid changed codon 84 serine to isoleucine (AGC-ATC). Analyses was done by BioEdit alignment editor v7.2.5.

the mutations at the codon ( 83 ser) of the GyrA gene and (84 ser) of ParC was done by Hinf1 restriction endonuclease digestion. PCR amplified GyrA gene product $(937 \mathrm{bp}$ ) of all the isolates (mutant 83ser: two bands 601 and 336 bp, non-mutant 83 ser: three bands 601, 200 and $136 \mathrm{bp}$ ), and ParC product (992 bp) of all the isolates (mutant 84ser: two bands 709 and $273 \mathrm{bp}$, non-mutant 84 ser: three bands 656, 273 and $53 \mathrm{bp}$ ) as seen on the agarose gel (Fig.2) all samples showed mutations at (serine 83) of GyrA and (serine 84) of ParC.

Sequencing of GyrA, GyrB, ParC and ParE Genes: Sequencing of all QRDR P. mirabilis, performed by alignment with reference strain $P$. mirabilis HI4320 in GenBank database NCBI by nucleotide blast revealed:

- Mutations at (codon 83) of gyrA possessed serine to isoleucine substitution (G $248 \mathrm{~T}$ ) this was observed in one strain (33.3\%) (Table-II and Fig.3).

- Mutations at codon 84 and silent mutation at codon 81 and 116 of ParC. Serine 84 to isoleucine $(66.6 \%)$ at resistant strains (1C and 3C) substitution (G $251 \mathrm{~T})$, codon 81 histidine $(33.3 \%)$ in sensitive strain (8C) substitution (C
$243 \mathrm{~T}$ ) and codon 116 proline (33.3\%) in resistant strain (3C) substitution (A $348 \mathrm{~T}$ ) (Table-II and Fig.4).

- Silent mutations at codons 474, 585, 612 and 639 of GyrB, Codon 474 leucine in all strains $(100 \%)$ including sensitive strain (8B) substitution (A $1422 \mathrm{G})$, codon 585 valine $(66.6 \%)$ in resistant strains (1B and 3B) substitution (T1 $755 \mathrm{C}$ ), codon 612 histidine $(33.3 \%)$ in sensitive strain (8B) substitution (C1836T) and codon 639 asparagine $(33.3 \%)$ in sensitive strain (8B) substitution (T1917C) (Table-II).

- Silent mutations at codons $(469,531$ and 533) of ParE, Codon (469) isolusine $(66.6 \%)$ in resistant stain (3E) and sensitive strain (8E) substitution (C $1407 \mathrm{~T})$, codon (531) aspartic acid (66.6\%) in resistant stain (3E) and sensitive strain (8E) substitution (C1593T) and codon (533) glycine $(33.3 \%)$ in sensitive strain (8E) substitution (1 599 A) (Table-II).

\section{DISCUSSION}

During the last decade, an increase in the incidence of fluoroquinolones resistance was reported among aerobic Gram negative bacilli. However, full 
Table-II: Accession numbers, ciprofloxacin susceptibility and QRDR mutations of Proteus mirabilis isolates.

\begin{tabular}{cccccc}
\hline \multirow{2}{*}{ Sample } & \multirow{2}{*}{ accession numbers } & Ciprofloxacin & \multirow{2}{*}{$\begin{array}{c}\text { Target } \\
\text { susceptibility }\end{array}$} & gene & Amino acid change \\
\cline { 5 - 6 } 1A & MH310924 & Resistance & GyrA & - & Nucleotide \\
3A & MH310925 & Resistance & GyrA & Ser 83 Ile & AGT-ATT \\
8A & MH310926 & Sensitive & GyrA & - & - \\
1B & MH310921 & Resistance & GyrB & Lus 474 Lus & TTA -TTG \\
& & & Val 585 Val & GTT -GTC \\
3B & MH310922 & Resistance & GyrB & Lus 474 Lus & TTA -TTG \\
& & & Val 585 Val & GTT -GTC \\
8B & MH310923 & Sensitive & GyrB & His 612 His & CAC-CAT \\
& & & Asn 639 Asn & AAT AAC \\
1C & MH310927 & Resistance & ParC & Ser 84 Ile & AGC-GTC \\
3C & MH310928 & Resistance & ParC & Ser 84 Ile & AGC-GTC \\
8C & MH310929 & Sensitive & Pro 116 Pro & CCA CCT \\
1E & MH310930 & Resistance & ParE & His 81 His & CAC CAT \\
3E & MH310931 & Resistance & ParE & Ile 469 Ile & ATC -ATT \\
& & & Asp 531 Asp & GAC-GAT \\
8E & MH310932 & Sensitive & ParE & Asp 531 Asp & GAC-GAT \\
& & & Glu 533 Glu & GGT-GGA \\
\hline
\end{tabular}

recognition of reports regarding the emergence of fluoroquinolone resistant $P$. mirabilis strains is still under investigation. Moreover, the genus Proteus is isolated from patients, especially from those with UTIs. $^{3}$

The results obtained from this study showed that $(30 \%)$ of $P$. mirabilis isolates were resistant to ciprofloxacin, this finding agrees with Rajivgandhi et al. from India ${ }^{10}$ and Kyung et al. from Korea ${ }^{11}$ who detected $(30 \%, 28 \%, 27 \%)$ of resistance of P. mirabilis resistance to ciprofloxacin, respectively. Different studies revealed different findings; in Sudan, Amir et al. found no resistance to ciprofloxacin in P. mirabilis. ${ }^{12}$ On the other hand, in Japan, lower percentage of resistance was found $(16 \%)^{13}$ while higher percentage was observed in Poland and in Taiwan; (40\% and 68.7\%), respectively..$^{14,15}$

Generally, the possible reasons behind the resistance to ciprofloxacin in Sudan may be the fact that this antibiotic have been in use for a long period and must have been abused, leading most properly to a change in the genome of the bacteria, making the target site of the antibiotic action inaccessible.
Important mechanisms of bacterial resistance to quinolone are the genetic mutations in the subunits GyrA and ParC of DNA gyrase and topoisomerase IV enzymes, as well as the subunits GyrB and ParE, which are also components of the target enzymes. ${ }^{13}$

P. mirabilis always mutate in GyrB (Ser 464 to Tyr or Phe), as mentioned by Saito et al..$^{13}$ This amino acid is not present in P. mirabilis GyrB sequence of clinical isolates but revealed silent mutations in the following codons; (474) leucine, (585) valine, (612) histidine and (639) asparagine. Also ParE gene always mutates in (Val 364 to Iso) in P. mirabilis, ${ }^{13}$ Thr-86-Ile, mutation from GyrA was the most common in Campylobacter jejuni. ${ }^{16}$ In this study, sequence analysis of ParE gene fragments from the clinical isolates revealed silent mutations in codons (469) isoleucine, (531) aspartic and (533) glycine. However, no mutations were detected in the corresponding region of pare neither in quinolone resistant nor in sensitive $P$. mirabilis isolates of this study. However, it is well known that ParE does not have an essential role in fluoroquinolone resistance among $P$. mirabilis as suggested previously. ${ }^{13}$ 
In this study, ciprofloxacin resistant $P$. mirabilis possessed mutations in GyrA (Ser 83 to Ile). This amino acid change is identical to those previously reported for fluoroquinolone resistance ${ }^{13}$ where $P$. mirabilis mutations in GyrA (Ser 83 to Arg or Ile) was proved. Other studies showed different mutations in GyrA with other bacteria; (Ser 80 to Leu 86) of Capnocytophaga spp. ${ }^{17}$, (Ser 83 to Leu) of E. coli ${ }^{18}$ and (Ser 83 to Phe) of M. bovis. ${ }^{19}$

P. mirabilis sequencing of $\operatorname{ParC}$ showed mutation in (Ser 84 to Ile) in this study. This result is in agreement with many researches who proved that Proteus mirabilis always mutated in GyrA (E87) and ParE (D420) for fluoroquinolone resistance. ${ }^{3}$ Also Edwardsiella tarda, another Gram negative bacillus, was found mutant in (Ser 84 to Ile) of $\operatorname{ParC}$ which is associated with fluoroquinolone resistance. ${ }^{5}$

In gram negative bacilli, fluoroquinolone resistance is mostly attributed to the antibiotic targets DNA gyrase and DNA topoisomerase IV structure change as the most significant mechanisms. ${ }^{20}$ In $E$. coli, resistance to ciprofloxacin may be obtained by more than two mutations in both GyrA and ParC genes. ${ }^{13,20}$ In this study however, only one or double mutation are enough for ciprofloxacin resistance in P. mirabilis.

In P. mirabilis, decreased susceptibility to fluoroquinolone is caused by mutations at residues (Ser 80 and Glu 84) of ParC of topoisomerase IV, a target of quinolones. ${ }^{13}$ Although both ParC and GyrA mutations are needed for acquisition of quinolones resistance ${ }^{13}$, in this study, one of the clinical isolates was found to have mutations only in $\operatorname{ParC}$ but not GyrA, which suggest that ParC is as important as GyrA in decreasing susceptibility to fluoroquinolones in P. mirabilis.

In contrast to the case of Acinetobacter baumannii, where silent mutation in QRDR regions were reported to be sufficient for fluoroquinolone resistance ${ }^{21}$, in $P$. mirabilis, sensitive strains in this study were found to possess silent mutation in GyrB, ParC and ParE.

Direct Hinf1 digestion of PCR product have been used by many researchers to screen GyrA and ParC genes mutations in different bacteria; S. pneumoniae at positions serine (83) of GyrA and serine (79) of $\operatorname{ParC}^{22}, A$. baumannii at positions gyrA (codons 83 and 87) and parC (codons 80 and $84)^{23}$ and N. gonorrhoeae at positions Ser (91) of GyrA ${ }^{24}$ were all found significantly associated with ciprofloxacin resistance.

In this study, direct Hinf1 digestion of PCR amplicons have been used to screen GyrA and ParC mutations in P. mirabilis. Mutations at codon (83) of the GyrA gene and (84) of ParC gene result in the loss of natural Hinfl site as identified. The results indicated that all resistant isolates mutated at serine (83) of GyrA and serine (84) of ParC. When dealing with sequencing, two out of the three sequenced GyrA appeared as non-mutated at Ser (83) while one sample of the other three sequenced $\operatorname{ParC}$ resulted as free from any mutations at ser (84). These findings may be attributed to the fact that serine (83) of GyrA and serine (84) of ParC in Proteus mirabilis consist of (AGC) which is almost different from serine in other bacteria (TCC). Thus the loss of natural Hinf1 site $\left(5^{\prime}\right.$...GANTC....3') will result.

In Sudan, many studies have been conducted to analyze antibiotic resistance in Gram-negative and Gram-positive bacteria isolated from different clinical specimens of both humans and animals. A wide range of resistance was detected which represent an alarm to the health authority of the country to take an action in order to control this phenomena. ${ }^{25}$

Limitations of the study: This research received no special fund, thus sequencing was performed to limited number of the isolates with random selection. Moreover, sample collection was limited only to the central teaching hospitals and the rural and terminal medical centers were not reachable.

\section{CONCLUSION}

In conclusion, direct Hinf1 digestion of PCR amplicons is not suitable to screen serine (83) of GyrA and serine (84) of ParC mutations in P. mirabilis. In addition, Proteus mirabilis ParC gene is as important as GyrA gene to cause ciprofloxacin resistance, with only one or two mutations in both GyrA and ParC genes of Proteus mirabilis being enough to obtain resistance to ciprofloxacin. Moreover, the study drew the attention of the clinicians to consider the percentage of resistance to Quinolones in order to try other options of treatment, that means drug susceptibility testing should be adopted to all patients of similar infections before starting a specific treatment.

Acknowledgments: Authors acknowledge the members and technical staff of the research laboratory of Sudan University of Science and Technology for their help and support. Special thanks to Dr. Hisham Altayeb for his valuable guidance and assistance.

Conflict of interest: None.

Funding: None 


\section{REFERENCE}

1. Schaffer JN, Pearson MM. Proteus mirabilis and Urinary Tract Infections. Microbiol spectrum, 2015;3(5). doi: 10.1128/microbiolspec.UTI-0017-2013

2. Endimiani A, Luzzaro F, Brigante G. Proteus mirabilis bloodstream infections: risk factors and treatment outcome related to the expression of extended-spectrum betalactamases. Antimicrob. Agents Chemother. 2005;49:2598 2605. doi: 10.1128/AAC.49.7.2598-2605.2005

3. Nakan R, Nakano A, Abe M, Nagano N, Asahara M, Furukawa T, et al. Prevalence and mechanism of fluoroquinolone resistance in clinical isolates of Proteus mirabilis in Japan. Heliyon, 2019;5(3):e01291. doi: 10.1016/j heliyon.2019.e01291

4. Kim J, Park Y, Kim SI. Nosocomial outbreak by Proteus mirabilis producing extended-spectrum beta-lactamase VEB-1 in a Korean university hospital. J Antimicrob Chemother. 2004;54:1144-1147. doi: 10.1093/jac/dkh486

5. Kim M, Lyu J, Soon B, Myoung A, Sung H, Kwangil K, et al. Mutations in the gyrB, parC, and parE Genes of Quinolone-Resistant Isolates and Mutants of Edwardsiella tarda. J Microbiol Biotechnol. 2010;20(12):1735-1743. PMID: 21193831

6. Elbadawi H, Elhag KM, Mahgoub E, Altayb HN, Hamid MMA. Antimicrobial resistance surveillance among gramnegative bacterial isolates from patients in hospitals in Khartoum State, Sudan. F1000Research. 2019;8:156. doi: 10.12688/f1000research.17744.1

7. CLSI 2010. Clinical and Laboratory Standards Institute. Performance standards for antimicrobial susceptibility testing. Twentieth informational supplement ed. CLSI document M100-S20. Wayne, PA

8. Kazuki H, Ayaka N, Takae S, Yujiro M, Ken K, Tadashi $\mathrm{M}$, et al. Phenotypic and molecular characterization of antimicrobial resistance in Proteus mirabilis isolates from dogs. J Med Microbiol. 2014;63:1561-1567. doi: 10.1099/ jmm.0.081539-0

9. SambrookJ, Fritsch EF, Maniatis T. (1989). Gelelectrophoresis of DNA. In: Molecular Cloning: a Laboratory Manual. New York: Cold Spring Harbor Laboratory Press, Cold Spring Harbor, NY, USA.

10. Rajivgandhi G, Maruthupandy M, Manoharan N. Detection of TEM and CTX-M genes from ciprofloxacin resistant Proteus mirabilis and Escherichia coli isolated on urinary tract infections (UTIs). J Microb Pathogen. 2018:123-130. doi: 10.1016/j.micpath.2018.05.024

11. Kyung M, Cheol-In K, Eun-Jeong J, Young E, Doo C, Kyong P, et al. Epidemiology of Ciprofloxacin Resistance and Its Relationship to Extended-Spectrum $\beta$ Lactamase Production in Proteus mirabilis Bacteremia. Korean J Intern Med. 2011;26(1):89-93. doi: 10.3904/kjim.2011.26.1.89

12. Amir S, Shadia AH, Magdi B, Salah S, Sultan A, Samir AA, et al. Elevated antibiotic resistance of Sundance urinary tract infection bacteria. EXCLI J. 2017;16:1073-1080. doi: 10.17179/excli2017-424

13. Saito R, Sato K, Kumita W, Inami N, Nishiyama H, Okamura $\mathrm{N}$, et al. Mutations of DNA gyrase and topoisomerase IV in clinical isolates of fluoroquinolone-resistant Proteus mirabilis. Jpn J Antibiot. 2006;59(1):41-43. PMID: 16673582

14. Joanna K, Krzysztof S, Katarzyna Z, Eugenia G. The Assessment of Proteus mirabilis Susceptibility to Ceftazidime and Ciprofloxacin and the Impact of These Antibiotics at Subinhibitory Concentrations on Proteus mirabilis Biofilmsl. Biomed. Res. Int 2013;2013:930876. doi: $10.1155 / 2013 / 930876$
15. Wang JT, Chen C, Chang S, Shiau Y, Wang H, Lai J, et al. Antimicrobial susceptibilities of Proteus mirabilis: a longitudinal nationwide study from the Taiwan surveillance of antimicrobial resistance (TSAR) program. BMC Infect Dis. 2014;14:486. doi: 10.1186/1471-2334-14-486

16. Mclver CJ, Hogan TR, White PA, Tapsall JW. Patterns of quinolone susceptibility in Campylobacter jejuni associated with different gyrA mutations. Pathology. 2004;36:166-169. doi: 10.1080/00313020410001672019

17. Ehrmann E, Jolivet-Gougeon A, Bonnaure-Mallet M, Fosse T. Role of DNA gyrase and topoisomerase IV mutations in fluoroquinolone resistance of Capnocytophaga spp. clinical isolates and laboratory mutants. J Antimicrob Chemother. 2017;72:2208-2212. doi: 10.1093/jac/dkx119

18. Varughese LR, Rajpoot M, Goyal S, Mehra R, Chhokar V, Beniwal V. Analytical profiling of mutations in quinolone resistance determining region of gyrA gene among UPEC. PLoS One 2018;13(1):e0190729. doi: 10.1371/journal. pone.0190729

19. Lysnyansky I, Mikula I, Gerchman I, Levisohn S. Rapid Detection of a Point Mutation in the parC Gene Associated with Decreased Susceptibility to Fluoroquinolones in Mycoplasma bovis. Antimicrob Agents Chemother. 2009;53:4911-4914. doi: 10.1128/AAC.00703-09

20. Bhatnagar $\mathrm{K}$, Wong A. The mutational landscape of quinolone resistance in Escherichia coli. PLoS One. 2019;14(11):e0224650. doi: 10.1371/journal.pone.0224650

21. Ardebili A, Rastegar LA, Beheshti M, Rastegar LE. Association between mutations in gyrA and parC genes of Acinetobacter baumannii clinical isolates and ciprofloxacin resistance. Iran J Basic Med Sci. 2015;18(6):623-626.

22. Stewart BA, Johnson AP, Woodford N. Relationship between mutations in parC and gyrA of clinical isolates of Streptococcus pneumoniae and resistance to ciprofloxacin and grepafloxacin. J Med Microbiol. 1999;48(12):1103-1106. doi: 10.1099/00222615-48-12-1103

23. Kakuta N, Nakano R, Nakano A,Suzuki Y, Tanouchi A, Masui $\mathrm{T}$, et al. A Novel Mismatched PCR-Restriction Fragment Length Polymorphism Assay for Rapid Detection of gyrA and parC Mutations Associated with Fluoroquinolone Resistance in Acinetobacter baumannii. Ann Lab Med. 2020;40(1):27-32. doi: 10.3343/alm.2020.40.1.27

24. Karim S, Bouchikhi C, Banani A, El Fatemi H, Souho T, Erraghay S, et al. Molecular Antimicrobial Resistance of Neisseria gonorrhoeae in a Moroccan Area. Infect. Dis. Obs. Gyne. 2018;2018:7263849. doi: 10.1155/2018/7263849

25. Abass AM, Ahmed ME, Ibrahim IG, Yahia SA. Bacterial Resistance to Antibiotics: Current Situation in Sudan. J Adv Microbiol. 2017;6(4):1-7. doi: 10.9734/JAMB/2017/36715

\section{Authors Contribution:}

RA: Specimen collection and molecular biology work.

ME: Approval of the proposal and general supervision, orientation and arrangement.

ME \& AY: Data analysis and manuscript writing and revision.

ME: Is responsible for the integrity of the entire work. All authors have contributed to this manuscript. 